\title{
Magnetic fields and radio polarization of barred galaxies
}

\section{D dynamo simulations}

\author{
K. Otmianowska-Mazur ${ }^{1}$, D. Elstner ${ }^{2}$, M. Soida ${ }^{1}$, and M. Urbanik ${ }^{1}$ \\ 1 Astronomical Observatory, Jagiellonian University, Kraków, Poland \\ 2 Astrophisikalisches Institut Postdam, Germany
}

Received 28 August 2001 / Accepted 5 December 2001

\begin{abstract}
A three-dimensional (3D) MHD model is applied to simulate the evolution of a large-scale magnetic field in a barred galaxy possessing a gaseous halo extending to about $2.8 \mathrm{kpc}$ above the galactic plane. As the model input we use a time-dependent velocity field of molecular gas resulting from self-consistent $3 \mathrm{D} N$-body simulations of a galactic disk. We assume that the gaseous halo rotates differentially co-rotating with the disk or decreasing its velocity in the $Z$ direction. The dynamo process included in the model yields the amplification of the magnetic field as well as the formation of field structures high above the galactic disk. The simulated magnetic fields are used to construct the models of a high-frequency (Faraday rotation-free) polarized radio emission that accounts for effects of projection and limited resolution, and is thus suitable for direct comparison with observations. We found that the resultant magnetic field correctly reproduces the observed structures of polarization $\boldsymbol{B}$-vectors, forming coherent patterns well aligned with spiral arms and with the bar. The process initializing a wave-like behavior of the magnetic field, which efficiently forms magnetic maxima between the spiral arms, is demonstrated. The inclusion of the galactic halo constitutes a step towards a realistic model of galactic magnetic fields that includes as many dynamical components as needed for a realistic description.
\end{abstract}

Key words. MHD - turbulence - galaxies: ISM - galaxies: magnetic fields

\section{Introduction}

Barred galaxies offer a unique opportunity to study the interrelations between gas flows and magnetic field structure. The bars are known to excite strong spiral patterns in the interstellar gas and to cause strongly nonaxisymmetric gas flows (Englmaier \& Gerhard 1997). Recent observations show that the magnetic field is strongly modified by gas shearing motions, although the field perturbations do not coincide with the suspected position of gas velocity perturbations, as expected from simple MHD models. Some concepts suggest that the nonaxisymmetric gas flows in the bar may constitute the main mechanism amplifying the galaxy-scale magnetic fields (Lesch \& Chiba 1997). Studying strong gaseous spiral patterns driven by the bar perturbation also offer a unique opportunity to understand the relationships between spiral arms and magnetic fields that are currently lively debated.

The question of the gas reaction to the bar potential has a long history (Moss et al. 1998; Moss et al. 1999 and references therein). However, little has been done to study the evolution of magnetic field in such objects.

Send offprint requests to: K. Otmianowska-Mazur, e-mail: otmian@oa.uj.edu.pl
Moss (1998) analyzed the magnetic field evolution in barred galaxies, however his models are restricted to two dimensions, while the vertical structures are essential for the magnetic field evolution. Regular magnetic fields are not only passively deformed by large-scale gas motions in the galactic plane but their evolution is also driven by turbulent motions via the dynamo mechanism. The latter process generates galaxy-scale poloidal (hence also vertical) magnetic field components. Though this genuinely three-dimensional magnetic field structure can still be analyzed in two dimensions by applying a solenoidality condition, we find it of great interest to apply a fully 3D model of the field evolution. Moreover we also get an essential vertical component of the large-scale gas motion, which requires a completely three-dimensional treatment. A threedimensional analysis of the interaction of the dynamo process with spiral arms has been performed by Rohde \& Elstner (1998) and Rohde et al. (1999). However, they considered only the turbulence enhancement in star-forming parts of spiral arms with no compression effects included, which is appropriate for the weak density wave objects (NGC 6946, Beck \& Hoernes 1996) they intended to explain. A comprehensive study of interactions of magnetic fields with bar-induced gas flows and spiral arms involving 
the dynamo process (hence carried out in three dimensions) is still urgently needed.

In our previous work (Elstner et al. 2000; von Linden et al. 1998; Elstner et al. 1998) we analyzed the magnetic field evolution under the influence of the bar and spiral arms. However, these studies assumed a thin gaseous disk only. A large gaseous halo may be of crucial importance for the problem. The dynamo process includes the generation of large-scale poloidal fields then transformed into azimuthal ones by differential rotation. A realistic analysis of the magnetic field evolution under a combined effect of the gas flows and the turbulent dynamo needs to involve an extended gaseous halo.

For these reasons we performed the study of magnetic field evolution assuming a galaxy composed of a disk and of a rarefied ionized halo with a scale height of $2.8 \mathrm{kpc}$. This expands our previous results involving a thin disk only. As previously, we use a realistic model of the gas flow, by analyzing motions of inelastically-colliding gas clouds in the self-consistent calculated gravitational potential of stars and gas. We adopted conditions suitable for the formation of a stellar bar. We assumed the magnetic field to be partially coupled to the gas via the turbulent diffusion process. We checked the results for all the assumptions by varying the diffusion coefficient, the strength of the dynamo action, and also by switching off the turbulent dynamo.

The magnetic field in external galaxies is usually studied by means of the radio polarization, yielding the view of the magnetic field integrated along the line of sight and convolved to a certain radio telescope beam. As our models yield complex three-dimensional magnetic field structures, we decided to analyze our magnetic field structures by simulating the polarization maps, which yields much more concise information, easy to compare with observations.

\section{The model}

\subsection{Numerical methods}

Our experiment consists of two basic steps: the threedimensional $N$-body, sticky particle calculations yielding a realistic gas velocity field and a magnetohydrodynamical (MHD) model of the magnetic field evolution using the gas flow picture from the first simulations (Elstner et al. 2000; von Linden et al. 1998; Otmianowska-Mazur et al. 1997). The model galaxy consists of two essential components: a collision-less self-gravitating disk composed of star clusters as well as highly inelastic colliding gas clouds moving in the gravitational potential of stars and gas, as well as an analytical contribution due to the bulge and halo. The code involves a sticky-particle cloud scheme as described by Combes \& Guérin (1985). In our model the clouds were assumed to represent the large-scale velocity field of the gas (see Otmianowska-Mazur et al. 1997 for the detailed model description). We modeled the magnetic field
Table 1. Input parameters for $N$-body model.

\begin{tabular}{ll}
\hline Model & \\
\hline Mass in $10^{10} M_{\odot}:$ & \\
- dark-halo $M_{\mathrm{h}}$ & 9.6 \\
- disk $M_{\mathrm{d}}$ & 7.2 \\
- gas $M_{\mathrm{g}}$ & 1.6 \\
- bulge $M_{\mathrm{b}}$ & 4.8 \\
- $\left(M_{\mathrm{d}}+M_{\mathrm{g}}\right) / M_{\mathrm{tot}}$ & 0.38 \\
Scale length in kpc: & \\
- dark halo & 15 \\
- bulge & 1.2 \\
- disk & 6 \\
\hline
\end{tabular}

evolution by analyzing numerically the time-dependent solutions of the dynamo equation:

$$
\partial \boldsymbol{B} / \partial t=\operatorname{rot}(\boldsymbol{v} \times \boldsymbol{B})+\operatorname{rot}(\alpha \boldsymbol{B})-\operatorname{rot}(\eta \operatorname{rot} \boldsymbol{B})
$$

where $\boldsymbol{B}$ is the magnetic induction, $\boldsymbol{v}$ is the large-scale velocity of the gas, $\alpha$ is the dynamo coefficient (a tensor in general, having a diagonal form in our case) describing the mean helicity of turbulent motions and $\eta$ is the coefficient of a turbulent diffusion.

Calculations of the magnetic field are done with a modified version of the ZEUS3D code (Stone \& Norman 1992) neglecting the action of the magnetic field on the mean gas flow. The effects of the magnetic diffusion as well as the term of the $\alpha$-effect are introduced directly into the code (von Linden et al. 1998; Elstner et al. 2000). We introduced the galactic halo of ionized gas extending to $2.8 \mathrm{kpc}$ above the galactic plane. This value was chosen due to computational constraints and is four times larger than the disk thickness used in our earlier works (von Linden et al. 1998; Elstner et al. 2000).

To analyze the general properties of the magnetic field we decided to use the simulated maps of polarized intensity, constructed on the basis of our results. We performed it in the way similar to that described in our previous works (e.g. Otmianowska-Mazur et al. 2000) namely, by integrating the Stokes parameters $I, Q$ and $U$ along the line of sight, convolving them with an assumed beam and computing the maps of polarized intensity and the angle of polarization $\boldsymbol{B}$-vectors. This process yields information averaged over the line of sight, hence more concise, and directly comparable with observations.

\subsection{Model assumptions and input parameters}

The clouds span the mass range between $5 \times 10^{2}$ and $5 \times 10^{5} M_{\odot}$ distributed in 10 mass-bins with logarithmic intervals. The disk of the galaxy consists of 38000 star clusters (about $2 \times 10^{6} M_{\odot}$ each) and 38096 clouds. The time step of calculations is $10^{7}$ years. The model parameters adopted in the computations are summarized in Table 1.

The kinematic evolution of the magnetic field is solved in a 3D rectangular grid of points, where the $X Y$ plane is 

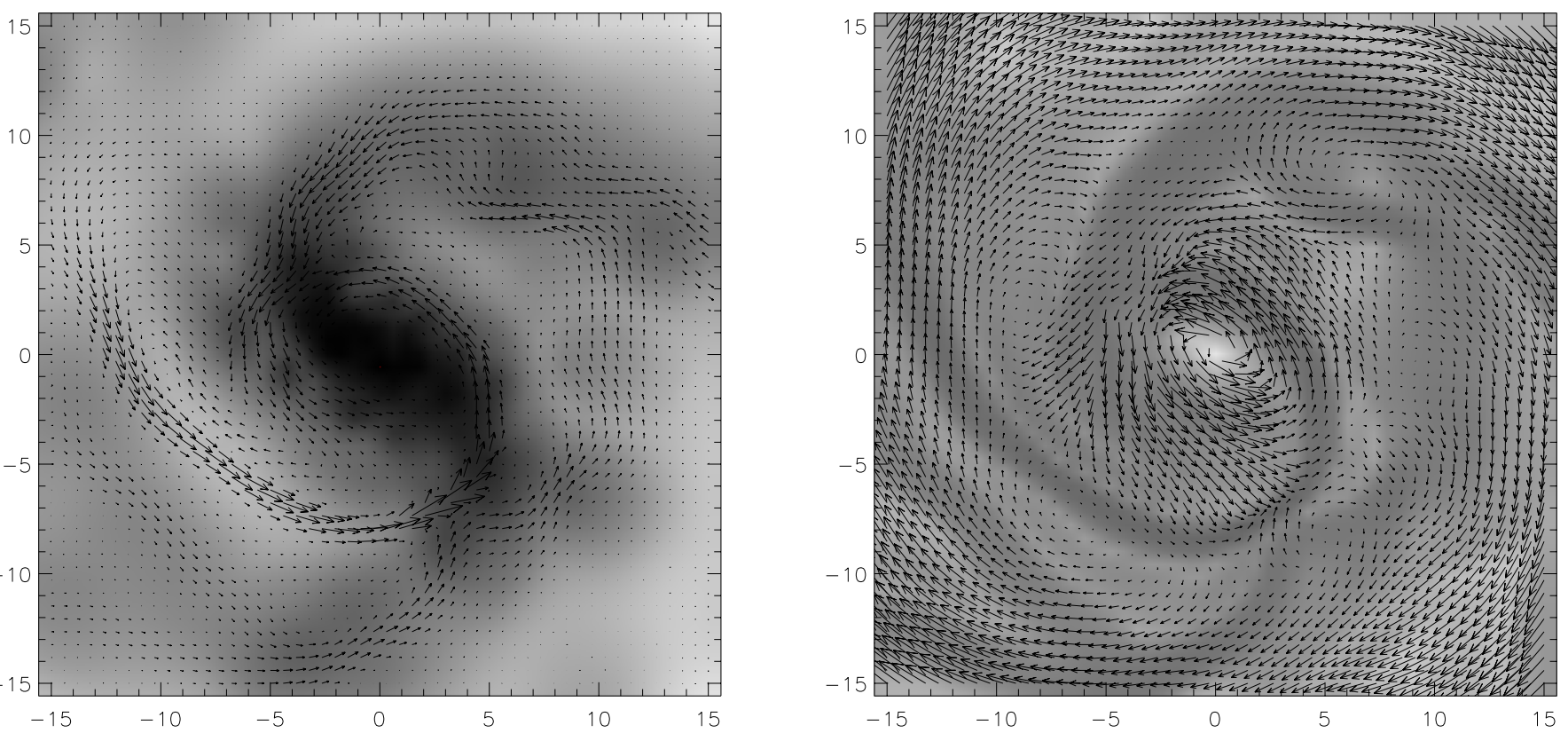

Fig. 1. Magnetic field and gas density in the midplane for model A1 at $0.35 \mathrm{Gyr}$ (left). Velocity vectors in the corotating frame with the bar and magnetic energy in greyscale at the midplane (right).

Table 2. Input parameters for MHD models.

\begin{tabular}{llllll}
\hline Model & A1 & A2 & A3 & B & C \\
\hline$\alpha_{0 x x}=\alpha_{0 y y}\left[\mathrm{~km} \mathrm{~s}^{-1}\right]:$ & 10 & 1.0 & 0.1 & 0 & 10 \\
$\alpha_{0 z z}\left[\mathrm{~km} \mathrm{~s}^{-1}\right]:$ & -10 & -1.0 & -0.1 & 0 & -10 \\
$\eta_{0}\left[\mathrm{~cm}^{2} \mathrm{~s}^{-1}\right]:$ & $3 \times 10^{26}$ & $3 \times 10^{25}$ & $3 \times 10^{25}$ & $3 \times 10^{26}$ & $3 \times 10^{26}$ \\
rotation of halo in $Z$ direction: & const. & const. & const. & const. & decreasing \\
\hline
\end{tabular}

the galactic plane and the $Z$ axis is the axis of rotation. For all experiments we use $0.3 \mathrm{kpc}$ as a grid step along the $X$ and $Y$ axes and $0.08 \mathrm{kpc}$ along the $Z$ axis. The disk scale height is $0.8 \mathrm{kpc}$, and halo height is $2.8 \mathrm{kpc}$ above the galactic plane. The number of grid points in $X$ and $Y$ directions is 101 , while in $Z, 71$ points. The adopted time step is $0.5 \times 10^{5} \mathrm{yr}$.

We performed simulations of magnetic field evolution in a barred gas-rich galaxy with five different sets of parameters. The input parameters for all models are summarized in Table 2. We include the diagonal components of the $\alpha$ tensor changing sinusoidally in the disk along the $Z$-direction with the maximum value of $\alpha_{0}$ presented in Table 2. In the halo we assume $\alpha_{x x}=\alpha_{y y}=\alpha_{z z}=0$ above $1 \mathrm{kpc}$. In order to saturate the dynamo process at equipartition field strength $B_{\text {eq }}$ we adopt the usual $\alpha$-quenching with $\alpha=\alpha_{0} /\left(1+\left(B / B_{\text {eq }}\right)^{2}\right)$ (Elstner et al. 2000). The diffusion coefficient $\eta$ grows linearly in the $Z$-direction, which is the result of the hydrostatic equilibrium assumption on the galactic disk (Fröhlich \& Schultz 1996). Its midplane value of $3 \times 10^{26} \mathrm{~cm}^{2} / \mathrm{s}$ results from a turbulent velocity of $10 \mathrm{~km} \mathrm{~s}^{-1}$ and a correlation time of $3 \times 10^{7}$ years. Due to uncertainty in the correlation time and its possible reduction by the magnetic fields we assume that the value of $\eta$ is ten times smaller in experiments A2 and A3 (without $\eta$-quenching). In A1-B models we apply differential rotation which is independent of the height above the disk plane up to $2.8 \mathrm{kpc}$. Such an assumption is based on the observational results derived by different authors that the Galactic halo co-rotates or nearly co-rotates with the disk up to $3-4 \mathrm{kpc}$ above the galactic disk (Kalberla \& Kerp 1998 and references therein). In model C the speed of the differential rotation in the halo decreases exponentially with increasing distance from the disk boundary to half of its disk value, according to recent observation of NGC 5775 (Tüllmann et al. 2000). In order to study possible changes of the magnetic field structure caused by the bar and spiral arms we start our simulations from an already-evolved magnetic field configuration computed using the initial velocity field.

The polarization maps at selected time steps of our models are constructed with the inclination of $30^{\circ}$ (almost face-on) and $85^{\circ}$ (almost edge-on). The model assumes the relativistic electrons with an energy spectral index $\gamma=2.8$. Their distribution is assumed to change vertically as a Gaussian with the scale-height of $1 \mathrm{kpc}$. Radially, the electron density is uniform up to $R=12 \mathrm{kpc}$. We adopt $70 \%$ as a maximum polarization degree of synchrotron 


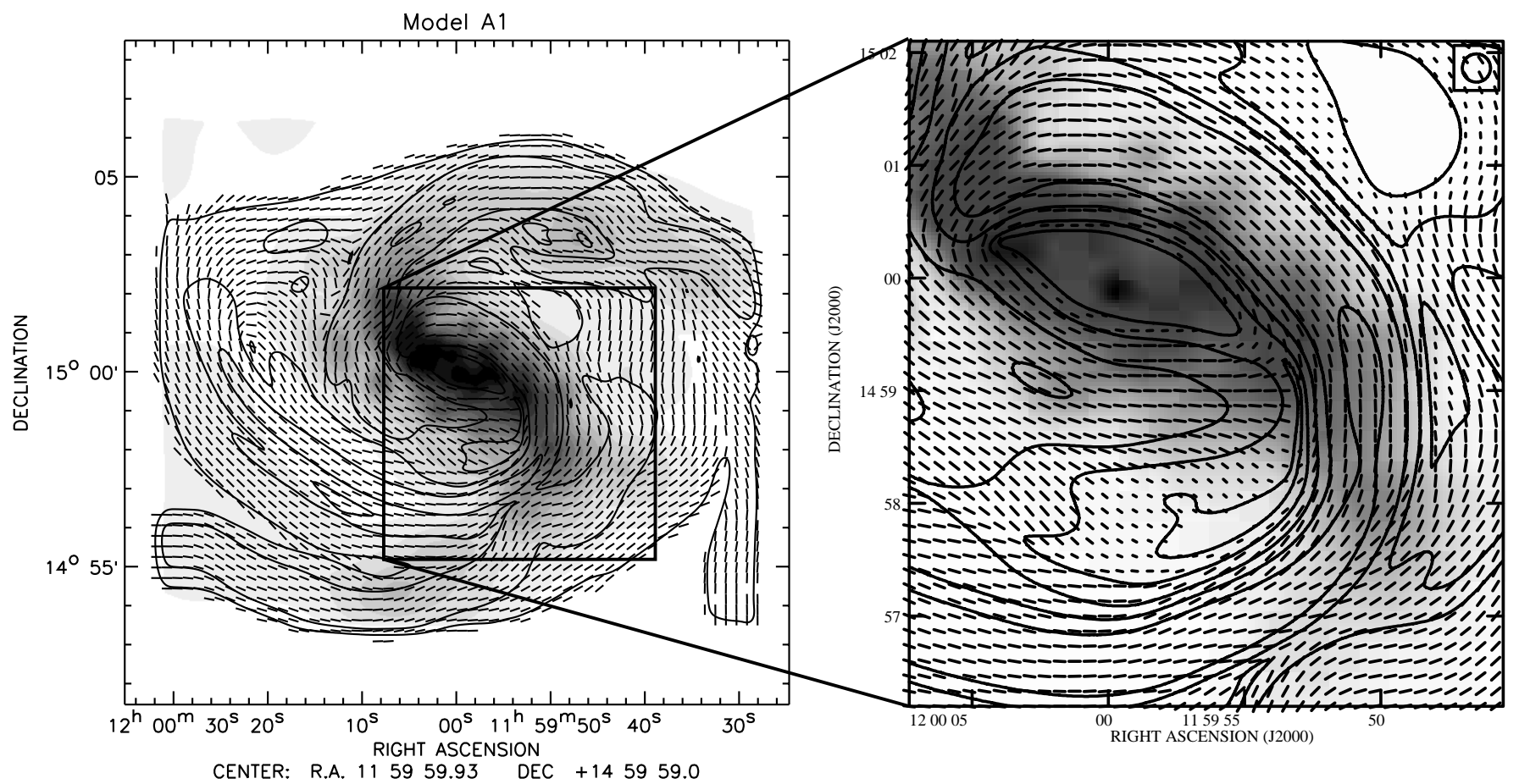

Fig. 2. Polarization map of model A1 at time $0.35 \mathrm{Gyr}$ and inclination of $30^{\circ}$ superimposed onto gaseous map (grey plot) integrated along the line of sight. Enlargement of the most interesting region is shown on the right.

radiation (Pacholczyk 1970; Otmianowska-Mazur et al. 2000 ) and neglect any Faraday and absorption effects. The maps are convolved with a beam of $10^{\prime \prime}$ and $15^{\prime \prime}$ for the edge-on $\left(85^{\circ}\right)$ and face-on $\left(30^{\circ}\right)$ inclination, respectively.

\section{Results}

\subsection{Magnetic field structure in selected planes}

The bar turns rigidly with a corotation radius of about 8 kpc. The gaseous spiral arms are excited by a bar perturbation but they follow the bar at a somewhat lower angular speed. They break into parts, some of them again join the opposite bar end while the others slowly disperse. This is accompanied by a continuous creation of new arms. The gas density maxima, being the regions of strongest cloud coagulation, can be identified with dust lanes accompanying spiral arms in galaxies. Shock fronts, which are seen in hydrodynamical gas simulations with a bar gravitational potential, could be identified with the gas density enhancements seen in our simulations, despite the limited resolution of our sticky particle model. The velocity in the corotating frame shows a very similar behavior to the hydrodynamical models with a strong change in velocity direction at the location where the shock is expected.

All calculated models yield qualitatively similar magnetic field structures, so we decided to present figures mainly for the model A1. The large-scale magnetic field quickly responds to non-axisymmetric velocity perturbations of the gas flow in the bar and spiral arms. The magnetic field is locally amplified by a local compression near leading sides of the bar and inner edges of the spiral arms.
It already forms magnetic arms at early stages of evolution. As the gaseous arms dissipate, the magnetic ones remain, often between newly formed gaseous arms. In Fig. 1 where magnetic vectors are superimposed onto the gas density grey plot at the time step of $3.5 \times 10^{8} \mathrm{yr}$, we can see the magnetic maxima related to newly created arms as well as those corresponding to arm segments present some time ago. The magnetic arm amplified at the earlier stages (about $100 \mathrm{Myr}$ ago) is visible in the south-west part of Fig. 1 (left). The pitch angles of the magnetic vectors in the interarm space are similar to those of gaseous arms. The time at which the results are presented in the figures was chosen so to provide the best resemblance to observations shown in Fig. 3.

In Fig. 1 (right) the velocity vectors (in a co-rotating frame) are superimposed onto the magnetic intensity grey plot showing the character of gaseous flow at the same time interval. In region near the southern end of the bar we can see that the radial velocity changes its sign, causing the amplification of the magnetic field there. Such regions are distributed along gaseous non-axisymmetric features at different time steps and they could be identified with gaseous shocks.

\subsection{The polarization maps}

The map of polarization vectors for a simulated almost face-on $\left(30^{\circ}\right)$ galaxy (model A1, see Table 2), superimposed onto the contours of polarized intensity obtained for the same time step $\left(3.5 \times 10^{8} \mathrm{yr}\right)$ as in Fig. 1, is presented in Fig. 2. The resultant distributions of polarized vectors and intensity look much smoother than the vectors 


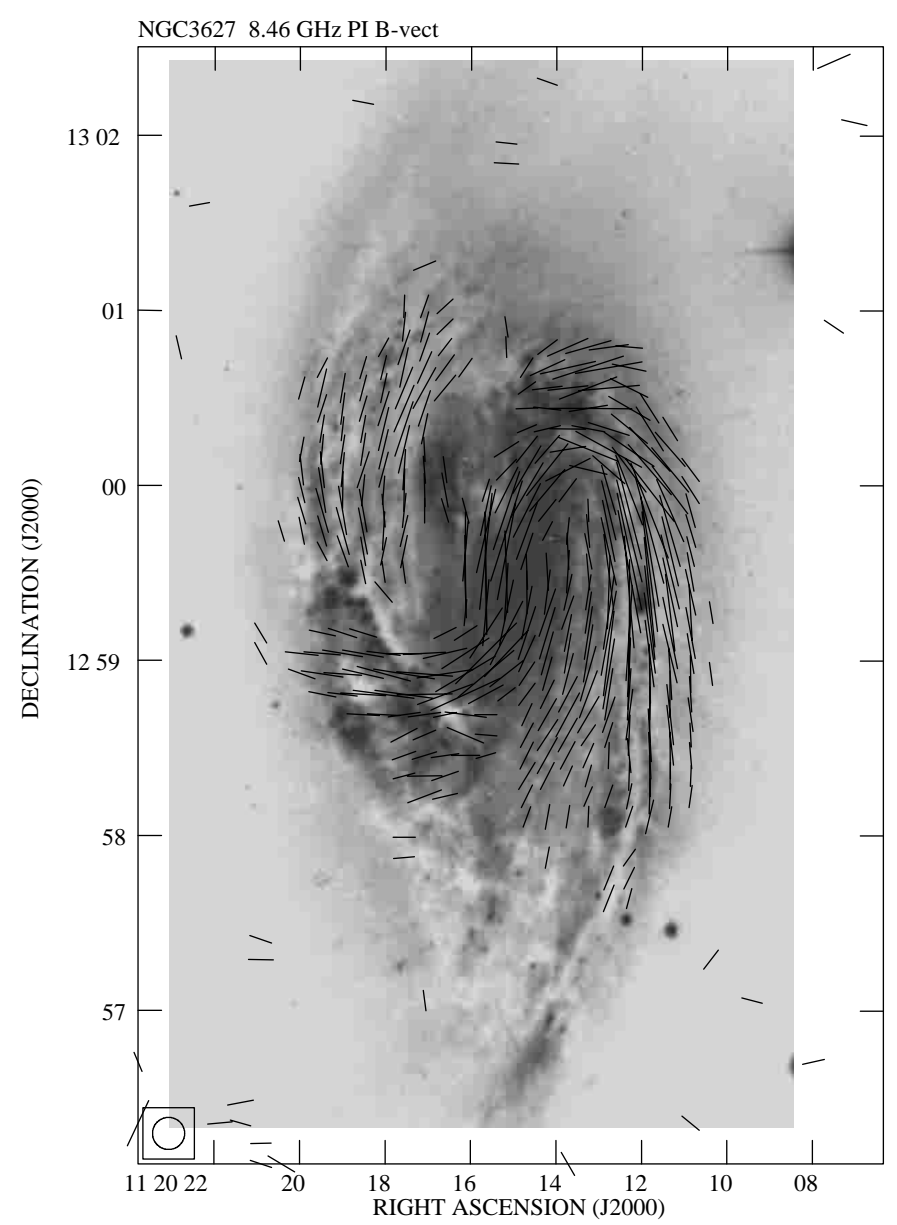

Fig. 3. The $\boldsymbol{B}$-vectors of length proportional to polarized intensity of NGC 3627 at $8.46 \mathrm{GHz}$ superimposed onto the optical image by Arp (1966). A vector length of $10^{\prime \prime}$ corresponds to a polarized intensity of $50 \mu \mathrm{Jy} / \mathrm{b}$.a. The orientations of vectors have been corrected for Faraday rotation effects. The map resolution is $11^{\prime \prime}$ (see Soida et al. 2001).

presented in Fig. 1, which is due to beam smoothing and integration of magnetic field structures along the line of sight. The most conspicuous features in these maps are the bright polarized arms extending along the gaseous spirals and also in the interarm regions. Figure 2 (left) presents a three-armed structure of polarized ridges. Two of them coincide with the gas density maxima constituting newly generated gaseous arms. Another one runs from the SW bar end to the interarm space towards the east and traces an older gaseous structure detached and dispersed 100 Myr ago. In this respect the magnetic arm may trace old gaseous patterns which were generated and faded away a long time ago in galactic history. In experiments with a high diffusion coefficient some polarized emission also fills smoothly the interarm space, as is often observed in spiral galaxies (Beck et al. 1996).

The polarization $\boldsymbol{B}$-vectors are generally aligned with gaseous spiral arms also in the interarm space, forming a coherent spiral pattern with pitch angles similar to what we identify as the dust lanes. However, in the model A1 the $\boldsymbol{B}$-vectors show locally strong pitch angle differences between the arms and the interarm regions. This is particularly visible for the region around $\mathrm{RA}_{2000}: 11^{\mathrm{h}} 59^{\mathrm{m}} 54^{\mathrm{s}}$; $\mathrm{Dec}_{2000}: 14^{\circ} 58^{\prime} 30^{\prime \prime}$ where the $\boldsymbol{B}$-vectors in the arm and the interarm meet at almost right angles (Fig. 2 (right)). The interarm vectors run parallel to the bar turning suddenly towards the orientation of a spiral arm, which resembles the situation in the barred galaxy NGC 3627 as presented in Fig. 3 (Soida et al. 2001). We note particularly the region around $\mathrm{RA}_{2000}: 11^{\mathrm{h}} 20^{\mathrm{m}} 14^{\mathrm{s}}$; $\operatorname{Dec}_{2000}: 13^{\circ} 00^{\prime}$ and that around $\mathrm{RA}_{2000}: 11^{\mathrm{h}} 20^{\mathrm{m}} 18^{\mathrm{s}} ; \operatorname{Dec}_{2000}: 12^{\circ} 59^{\prime}$ in Fig. 3 where the arm and interarm $\boldsymbol{B}$-vectors meet at right angles. We note also that in Fig. 2 around the region of $\mathrm{RA}_{2000}$ : $11^{\mathrm{h}} 59^{\mathrm{m}} 55^{\mathrm{s}}$; Dec 2000 : $14^{\circ} 59^{\prime} 30^{\prime \prime}$, the $\boldsymbol{B}$-vectors turn suddenly from the orientation perpendicular to the bar to that parallel to the bar. This bears some (limited) resemblance to the situation in NGC 1097 (Beck et al. 1999). We note however that the latter galaxy shows a much stronger bar than that in our model. For this reason the validity of such a comparison is still limited and will be a subject of a separate study involving an analytically introduced bar potential.

The bright polarized arms can be conveniently analyzed in the frame of azimuthal angle in the disk with respect to the bar orientation and $\ln (r)$, in which a logarithmic spiral appears as a straight line inclined by its pitch angle. We analyze both the position of the arms relative to the bar and the orientations of polarization $\boldsymbol{B}$-vectors. Figure 4 shows the time evolution of the model B at four time steps: $2.3,2.5,2.7$ and $2.9 \times 10^{8} \mathrm{yr}$. A greyplot of the gas density (logarithmic scale) is included, as well. In our frame the gaseous structures in the inner disk (thus more directly connected to the bar) do not change their position much. However, weak gas density maxima in the outer disk $(r>8 \mathrm{kpc})$ systematically drift to the left in Fig. 4, being thus delayed with respect to the bar rotation. The inner parts of the magnetic arms remain tied to the gaseous structures and do not change the position much with respect to the bar. An obvious drift of magnetic structures occurs in the outer disk (Fig. 4). A kind of "magnetic wave" move to the left, being continuously generated at the bar ends.

In order to compare the time evolution of pitch angles of polarization $\boldsymbol{B}$-vectors obtained for all models, we averaged the pitch angle values between the radius $r=8 \mathrm{kpc}$ and $12 \mathrm{kpc}$ along the azimuthal direction for all time steps (Fig. 5). For all models the absolute value of the pitch angle is quite high - from about $18^{\circ} \pm 2.6^{\circ}$ for model $\mathrm{B}$ (without the dynamo mechanism) to about $22^{\circ} \pm 2.5^{\circ}$ for our base model (A1). For the simulations with smaller dynamo coefficients after 300 Myr (model A2) the mean pitch angle grows with time from $-17^{\circ}$ to about $-22^{\circ}$ with a std. dev. of $\pm 4^{\circ}$.

Figure 6 presents the structure of the poloidal magnetic field at the disk/halo interface for the evolution time of 490 Myr for model A1 in the plane perpendicular to the galactic plane. Magnetic structures in the halo are formed quite quickly and they persist over most of the evolution time. The excited mode is mainly of an S0 type, 

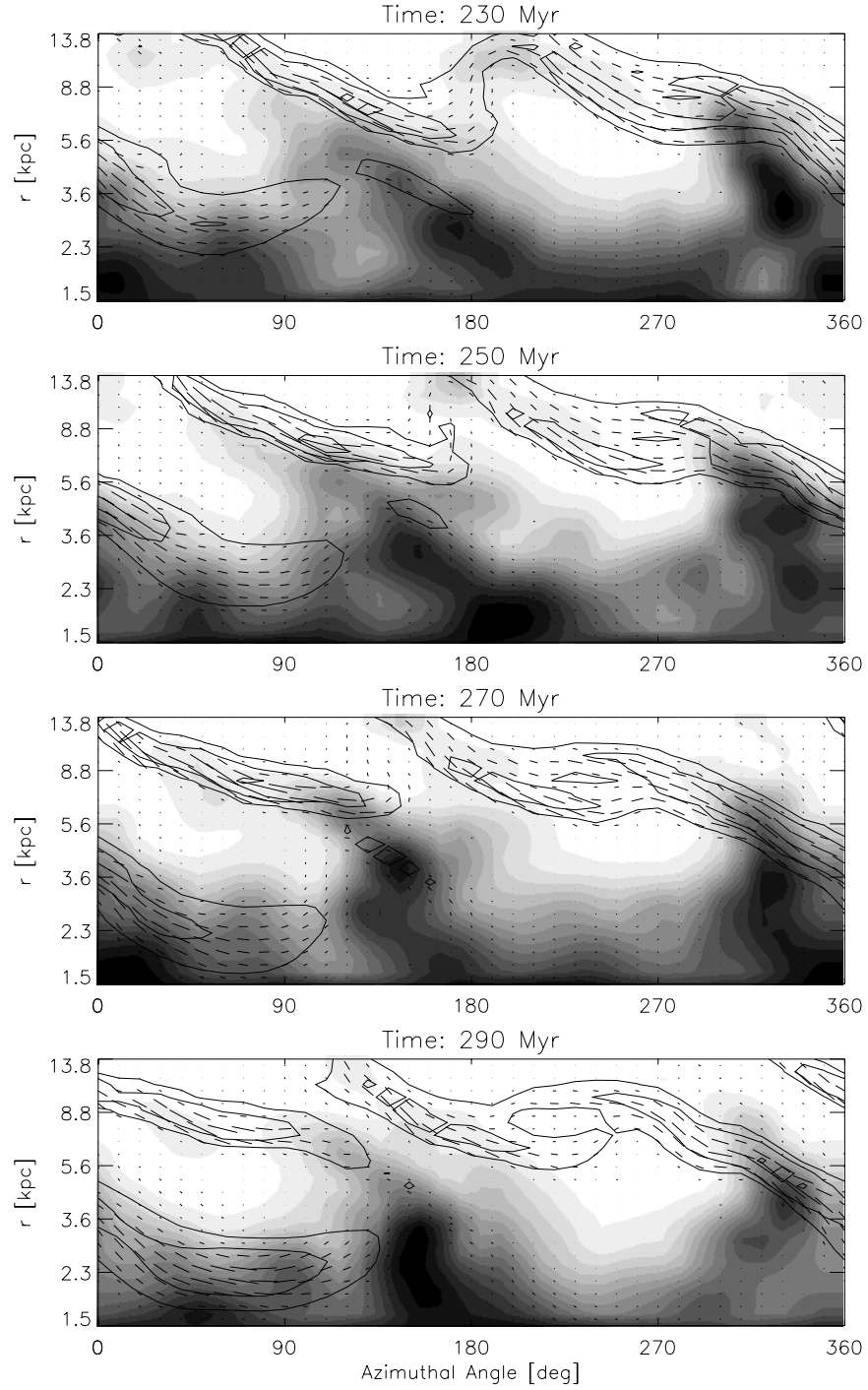

Fig. 4. Contour plots of polarized intensity maps and $\boldsymbol{B}$-vectors from model B superimposed onto the gas density maps (integrated along the line of sight) shown in the azimuth $\log (r)$ frame at four time steps.

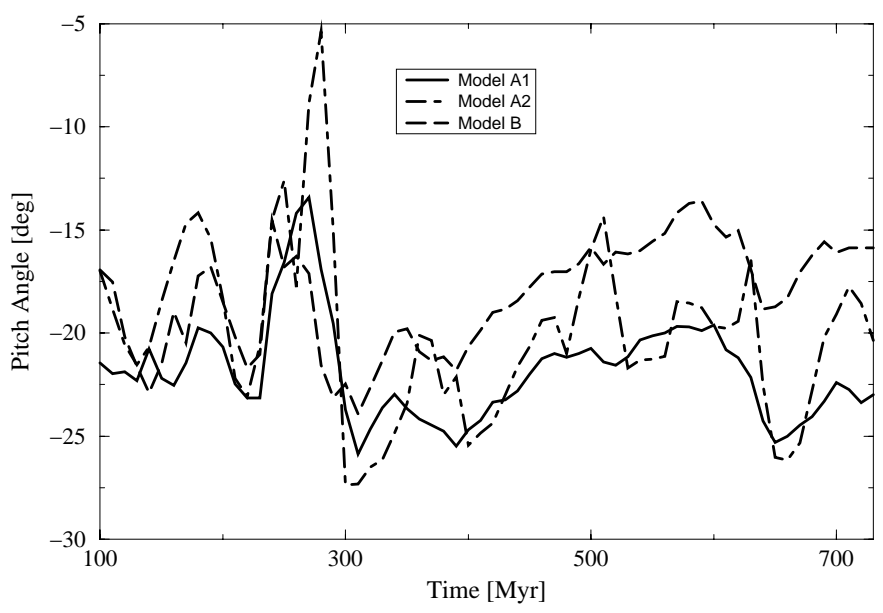

Fig. 5. The evolution of mean magnetic pitch angle for models A1, A2 and B, averaged in galactocentric rings between 8 and $12 \mathrm{kpc}$.

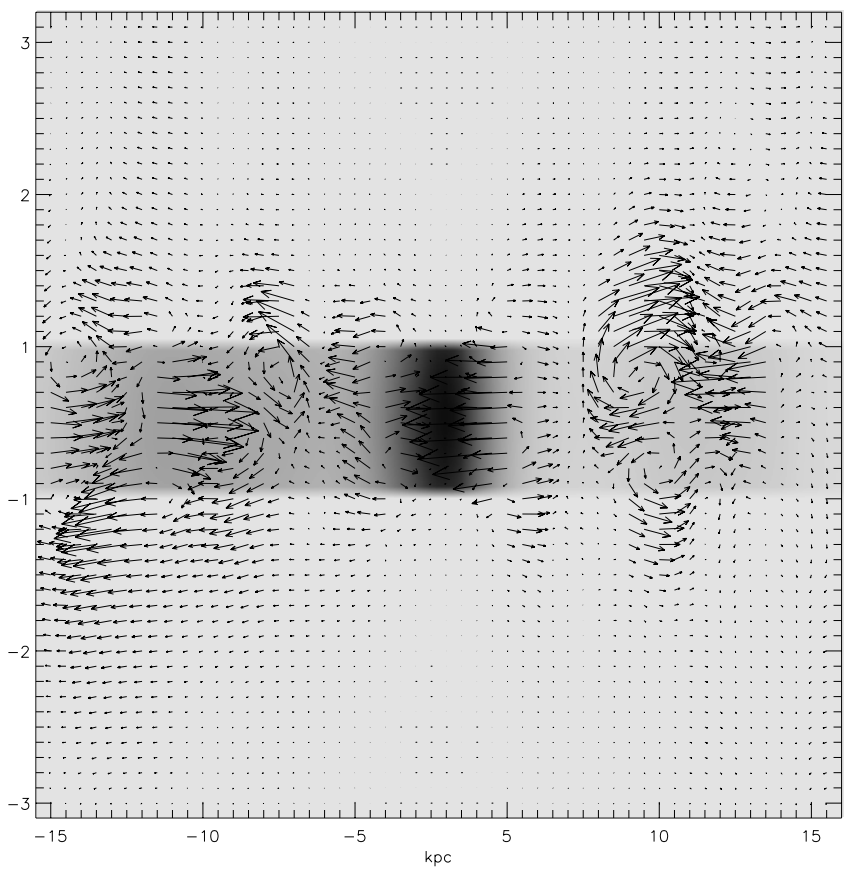

Fig. 6. The magnetic field vectors and gas density shown in the plane perpendicular to the galactic plane at time step $490 \mathrm{Myr}$.

which is a consequence of the chosen form of the dynamo tensor $\alpha$. In Fig. 6 we can see that the magnetic lines of force form separate loops under and above the galactic plane, having opposite senses of circulation. Instead of single, galaxy-scale loop-like structures we obtained rather smaller, localized structures centered at various heights up to $1.5 \mathrm{kpc}$ above the disk plane. The growing value of the magnetic diffusion in the halo does not allow the formation of stronger magnetic structures higher than $2 \mathrm{kpc}$ above the disk plane. The calculations with the decreasing rotational velocity (model C, see Table 2 ) in the halo give no significant differences between models having the same value of the other dynamo parameters (Brandenburg et al. 1993).

Figure 7 presents the edge-on view of resultant polarization $\boldsymbol{B}$-vectors overlaid onto the polarization intensity contours projected almost edge-on (inclination $=85^{\circ}$ ) onto the sky for the model A1 at the time step 510 Myr. The polarization map shows the vectors which are parallel to the galactic disk. This is caused by the fact that we see only the parts of poloidal loops closest to the plane where there are enough cosmic ray electrons to generate significant synchrotron emission. The projection and beam smoothing effects efficiently blur all localized vertical fields leaving only its mean plane-parallel component seen in polarization. This resembles the majority of galaxies (except NGC 4631, Golla \& Hummel 1994), in which a disk-parallel magnetic field is observed close to the disk plane (Dumke et al. 1995; Dumke et al. 2000; Beck et al. 1996).

Figure 8 shows the magnetic energy evolution for the models A1, A3 and B. We started our simulations with an already evolved dynamo magnetic field rather than with 


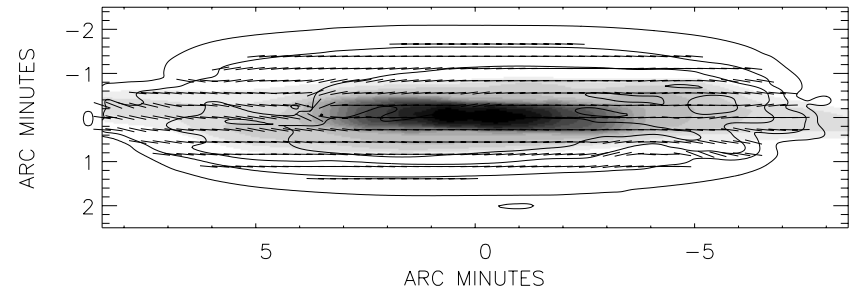

Fig. 7. The edge-on (inclination of $85^{\circ}$ ) polarized intensity map with $\boldsymbol{B}$-vector map from model A1 at time 510 Myr. $1^{\prime}$ corresponds to about $2 \mathrm{kpc}$ in the modeled galaxy.

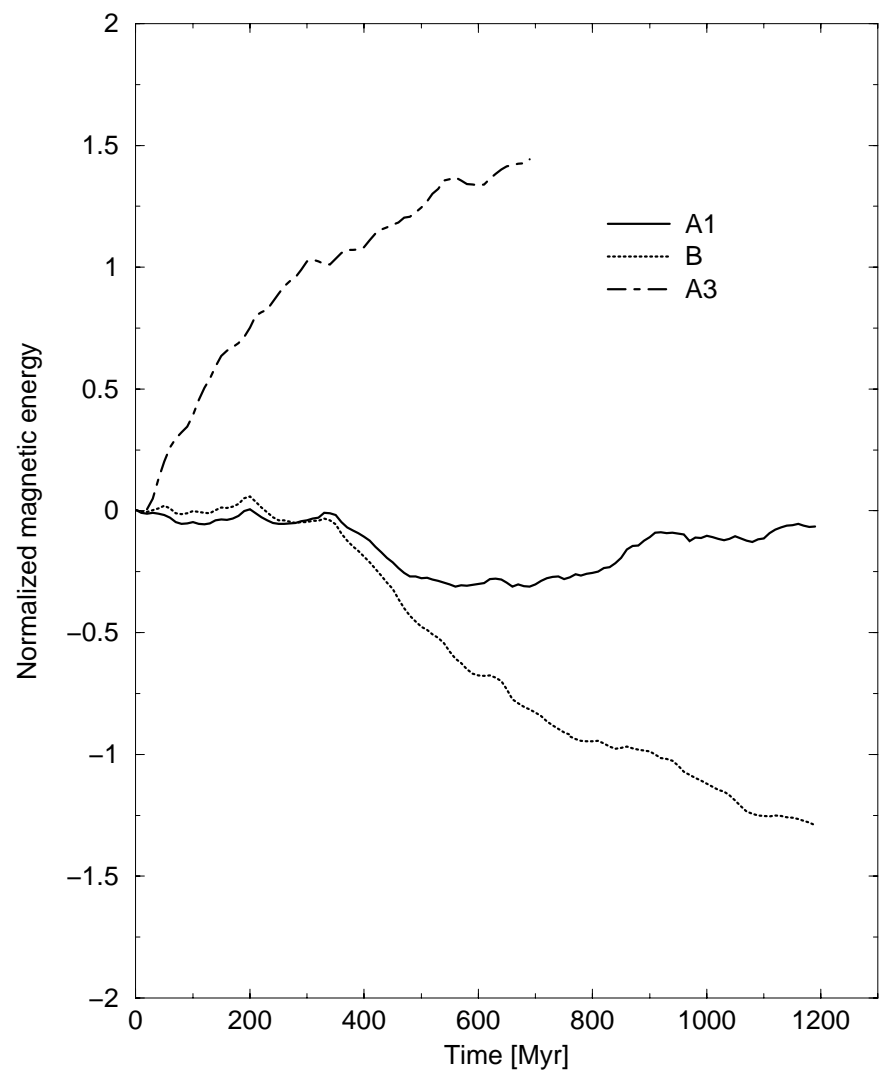

Fig. 8. Time evolution plots of the total magnetic energy normalized to the initial value (in logarithmic scale) for models A1, A3 and B. The initial field was the saturated field calculated with the primary velocity for the parameters of model A1.

a weak seed field, therefore the energy of the model A1 remains nearly constant. The magnetic energy of model B with the same diffusivity decreases, because no further turbulent dynamo action occurs. Surprisingly, the magnetic energy of model A3 with a very small value of $\alpha$ and with reduced turbulent diffusivity is increasing. The dynamo number has the same value as in model A1. Therefore the magnetic energy should remain constant, similar to model A1. The only explanation for the growth is an amplification by the velocity. At that point it is difficult to decide if the velocity itself is acting as a dynamo. Because of a nearly linear growth in the magnetic field strength (square root of the energy) it could be a temporary increase of magnetic energy due to the shear flow. The vertical diffusion time for that model is about $10 \mathrm{Gyr}$. A consequence would be growth of the magnetic field without a major influence of $\alpha$. A quenched $\alpha$ and $\eta$ would give a further increase of magnetic energy, due to complicated 3D velocity flows. The surprise is that the pitch angle remains large even with a weak influence of $\alpha$. Further investigation of the magnetic field evolution in a lower diffusivity medium will be presented in a forthcoming paper.

\section{Discussion}

Our experiments clearly indicate that a strong magnetic component associated with spiral arms persists in spite of introducing the large gaseous halo and of the fact that spiral arm perturbations occur only in a thin disk.

The most interesting result is the fact that during the whole evolution time the modeled polarized intensity forms wave-like maxima apparently created in the compresion regions in the disk, seen as the gaseous arms. These gaseous spirals do not corotate with the bar but have a somewhat smaller angular velocity. This result is in agreement with earlier numerical works that proposed spiral arms having a pattern speed smaller than that of a bar (e.g. Sellwood \& Sparke 1988; Lindblad et al. 1996; Sellwood \& Wilkinson 1993; Tagger et al. 1987).

The most astonishing result is that the magnetic spiral pattern rotates slightly slower than the gaseous spirals almost independent of the diffusivity. This is a completely new result. This process causes a drift of magnetic field maxima from the gaseous spirals to the interarm space. This bears some quantitative resemblance to e.g. NGC 6946 (a weakly barred galaxy, Beck \& Hoernes 1996). Due to this mechanism magnetic arms could work as a tracer of spiral arms present at earlier time stages. For the first time we obtained rapid turning of the magnetic pitch angle between arm and interarm regions, which is also observed in NGC 3627 (Soida et al. 2001) and in NGC 1365 (Shoutenkov et al. 2000; Beck et al. in prep.; Lindblad 1999). This gives the impression of two separate components, one in spiral arms and one between them, as suggested by some observers (Soida et al. 2001). Simulations with different halo-to-disk mass ratios, resulting in a spiral pattern without a bar, did not show any difference between magnetic and optical pattern speeds (Elstner et al. 2000).

In contrast to our previous works the resultant magnetic field structure is fully three-dimensional with poloidal loops extending into the halo. Their existence does not contradict observations showing disk-parallel polarization vectors close to the disk plane (Dumke et al. 1995; Dumke et al. 2000). We note however a limited applicability of our model to particular real galaxies as we did not introduce concrete values of parameters to reproduce particular observations. We restrict ourselves to mentioning phenomenological features which may be worth further detailed studies to explain the observations in more detail. 


\section{Summary and conclusions}

The evolution of large-scale galactic magnetic fields in a galaxy with a gaseous halo and a bar has been demonstrated using 3D numerical simulations. In order to solve the dynamo equation we used velocities obtained from a self-consistent $N$-body, sticky particle code. We found that:

1. The magnetic arms persist in the presence of a large halo enabling a large-scale three-dimensional field.

2. The magnetic arms are also present in interarm regions, in agreement with observations.

3. Our calculations confirm the fact, known from earlier papers, that the pattern speed of the bar is higher than the pattern speed of spiral arms.

4. The magnetic field may take the form of a "magnetic wave" with magnetic arms drifting into the interarm areas due to dynamical dissipation and shear amplification independent of the value of the turbulent diffusion.

5. The magnetic pitch angles for all models keep the mean value between $-15^{\circ}$ and $-25^{\circ}$ independent of the diffusivity.

6. The edge-on view of the modeled magnetic field agrees with the majority of real observations.

Acknowledgements. The authors express their gratitude to Dr. Susanne von Linden for kindly supplying the velocity fields and to Dr. Anvar Shukurov for his valuable comments. This work was partly supported by a grant from the Polish Committee for Scientific Research (KBN), grant No. 4264/P03/99/17 and computing grant $\mathrm{KBN} / \mathrm{SPP} / \mathrm{UJ} / 011 / 1996$.

\section{References}

Arp, H. 1966, ApJS, 14, 1

Beck, R., \& Hoernes, P. 1996, Nature, 379, 47

Beck, R., Brandenburg, A., Moss, D., Shukurov, A. M., \& Sokoloff, D. D. 1996, ARA\&A, 34, 155

Beck, R., Ehle, M., Shoutenkov, V., Shukurov, A., \& Sokoloff, D. D. 1999, Nature, 397, 324

Brandenburg, A., Donner, K. J., Moss, D., et al. 1993, A\&A, 271,36
Combes, F., \& Gérin, M. 1985, A\&A, 150, 327

Dumke, M., Krause, M., Wielebinski, R., \& Klein, U. 1995, A\&A, 302, 691

Dumke, M., Krause, M., \& Wielebinski, R. 2000, A\&A, 355, 512

Elstner, D., Lesch, H., von Linden, S., Otmianowska-Mazur, K., \& Urbanik, M. 1998, Studia Geoph. Geod., 42, 373

Elstner, D., Otmianowska-Mazur, K., von Linden, S., \& Urbanik, M. 2000, A\&A, 357, 129

Englmaier, P., \& Gerhard, O. 1997, MNRAS, 287, 57

Fröhlich, H.-E., \& Schultz, M. 1996, A\&A, 311, 451

Golla, G., \& Hummel, K. 1994, A\&A, 284, 777

Kalberla, P. M. W., \& Kerp, J. 1998, A\&A, 339, 745

Lesch, H., \& Chiba, M. 1997, Fund. Cosmic Phys., 18, 273

Lindblad, P. A. B., Lindblad, P. O., \& Athanassoula, E. 1996, A\&A, 313, 65

Lindblad, P. A. B. 1999, A\&A Rev., 9, 221

von Linden, S., Otmianowska-Mazur, K., Lesch, H., \& Skupniewicz, G. 1998, A\&A, 333, 79

Moss, D. 1998, MNRAS, 297, 860

Moss, D., Korpi, M., Rautiainen, P., \& Salo, H. 1998, A\&A, 329, 895

Moss, D., Rautiainen, P., \& Salo, H. 1999, MNRAS, 303, 125

Otmianowska-Mazur, K., von Linden, S., Lesch, H., \& Skupniewicz, G. 1997, A\&A, 323, 56

Otmianowska-Mazur, K., Chyży, K., \& Soida, M. 2000, A\&A, 359, 29

Pacholczyk, A. G. 1970, Radio Astrophysics. Nonthermal Processes in Galactic and Extragalactic Sources (W. H. Freeman Co., San Francisco)

Rohde, R., \& Elstner, D. 1998, A\&A, 333, 27

Rohde, R., Beck, R., \& Elstner, D. 1999, A\&A, 350, 423

Sellwood, J. A., \& Sparke, L. S. 1988, MNRAS, 231, 25

Sellwood, J. A., \& Wilkinson, A. 1993, Rep. Prog. Phys., 56, 173

Soida, M., Urbanik, M., Beck, R., Wielebinski, R., \& Balkowski, C. 2001, A\&A, 378, 40

Shoutenkov, V., Beck, R., Shukurov, A., \& Sokoloff, D. 2000, in The Origins of Galactic Magnetic Fields, 24th meeting of the IAU, Joint Discussion 14, August 2000 (Manchester, $\mathrm{UK}), 34$

Stone, J. M., \& Norman, M. L. 1992, ApJS, 80, 791

Tagger, M., Sygnet, J. F., Athanassoula, E., \& Pellat, R. 1987, ApJ, 318, L43

Tüllmann, R., Dettmar, R.-J., Soida, M., Urbanik, M., \& Rossa, J. 2000, A\&A, 364, L36 